\title{
Aktuelle Methoden und Interventionen für die kreativtherapeutische und tanz-, bewegungstherapeutische Behandlung von Trauma bei Kindern und Erwachsenen
}

\author{
Iris Bräuninger
}

Dien

e Behandlung komplexer Traumafolgestörungen mit dissoziativen Störungen stellt trotz der Fülle an traumaorientierten Psychotherapie-Methoden eine besondere Herausforderung dar, die bislang kaum empirisch validiert wurde (Sack/ Sachsse 2013). Dies spiegelt sich auch in der geringen Evidenzbasierung traumaadaptierter körperpsychotherapeutischer und tanz-, bewegungstherapeutischer Ansätze wider. Im Mittelpunkt dieses Beitrages stehen eine Meta-Synthese qualitativer Ergebnisse zur Tanz-, Bewegungstherapie (Levine/Land 2015), Methoden und Interventionen zu Kreativen Therapien in der Traumabehandlung von Kindern (van Westrhenen/ Fritz 2014) sowie tanz-, bewegungstherapeutische Interventionen für die Bearbeitung von Dissoziation (Jorba-Galdos 2014; Pierce 2014) und Trauma (Dunphy et al. 2014; Pierce 2104) bei Erwachsenen.

\section{Qualitative Ergebnisse einer Meta-Synthese über Tanz-, Bewegungs- therapie und Trauma}

Die qualitative Meta-Synthese von Levine und Land (2015) generierte aus neun qualitativen Studien Erkenntnisse zu auftauchenden Themen und fasste ähnliche Ergebnisse zusammen. Die Autorinnen schlussfolgerten, dass in der Trauma-Behandlung vier Themen enthalten sein müssen:

1. Die Schaffung von Bewusstsein der Geist-Körper-Verbindung

2. Die Erweiterung des Bewegungsrepertoires (um Wirksamkeit, Empowerment und die Rückeroberung des Körpers zu unterstützen)

3. Die Schaffung einer neuen heilsamen Beziehung zum Selbst, zum Therapeuten / zur Thera- peutin oder zur Gruppe durch den Bewegungsprozess

4. Die Schaffung einer neuen gesunden Beziehung zur Bewegung.

Als Einschränkung der qualitativen Meta-Analyse wurde die kleine Anzahl an eingeschlossenen Studien erwähnt, von denen sich keine auf die Behandlung erwachsener Männer als Einzelklienten bezog.

\section{Kreative Therapien in der Behandlung traumatisierter Kinder}

Der Review von van Westrhenen und Fritz (2014) überprüfte 38 Studien zu Kreativen Therapien in der Behandlung traumatisierter Kinder auf Methodik, Population und theoretischen Rahmen. Der Schwerpunkt lag dabei auf der Zuverlässigkeit, Gültigkeit und Vertrauenswürdigkeit der Interventionen. Dreiviertel der Studien verwendeten ein qualitatives Design, was auch auf die drei eingeschlossenen Tanz-, Bewegungstherapiestudien zutraf (zwei Fallbeschreibungen, eine vorexperimentelle Studie). Die Ergebnisse zeigten, dass die Mehrzahl der Studien die Ergebnisse in prozessorientierter, narrativer Form präsentierten. Dies wurde von den Autorinnen kritisch und in Bezug auf die Interventionszuverlässigkeit als einschränkend bewertet. Die Autorinnen interpretierten die methodischen Schwächen von Studien der letzten zwölf Jahre als Grund, weshalb die wissenschaftliche Fundierung Kreativer Therapien hinter anderen beliebten Therapieansätzen zurückfalle. Als Lösung schlugen sie für die Zukunft eine engere Zusammenarbeit zwischen Forschern und Kreativtherapeuten vor, um einen höheren Forschungsstandard zu etablieren und 
umfassende, theoriegeleitete Rahmenbedingungen für die Behandlung traumatisierter Kinder zu entwickeln.

\section{Tanz-, bewegungstherapeutische Interventionen bei Dissoziation und Attachment-Trauma von Erwachsenen}

Jorba-Galdos (2014) ging von der Hypothese aus, dass eine Verbindung zwischen Kreativität und der Entwicklung von Compartmentalization (einer Form der Dissoziation mit der Unfähigkeit, „prinzipiell bewusst kontrollierbare oder bewusstseinsfähige mentale Prozesse angemessen zu steuern.“ Siehe Spitzer et al. 2015, 28) bestünde. Aufbauend auf dieser Hypothese stellte sie kreative Interventionen vor, welche die Minimierung und Entstehung falscher Erinnerungen verhindern sollten. Immobilität sei eine gängige Reaktion auf Trauma, weshalb KlientInnen zu Beginn der Therapie im ersten Schritt mehr Unterstützung beim Explorieren von Bewegung benötigten, beispielsweise durch Beobachten und Imitieren der Bewegungen des Therapeuten oder der Therapeutin. Sobald Bewegungsausdruck toleriert werden würde, könne im zweiten Schritt das Bewegungsrepertoire erweitert werden. Eine der wichtigsten Ziele in der Behandlung von Compartmentalization sei die Bearbeitung des ursprünglich traumatischen Ereignisses mit dem Ziel, Retraumatisierung zu verhindern und Integration zu fördern. Tanz, Bewegung und Improvisation könnten eine zentrale Rolle spielen, Wechsel zu ermöglichen und innere Systeme und Erinnerungen zu identifizieren. Grounding-Übungen könnten der Gefahr einer Überstimulierung entgegenwirken. Choreographische Arbeit und Performance eigneten sich, Übergänge zu meistern, Spaltungen aufzuheben und Erlebnisse auf affektiver, kognitiver, körperlicher sowie Wahrnehmungs- und Verhaltensebene zu integrieren.

Der vierte Beitrag beschreibt ein Modell tanz-, bewegungstherapeutischer Behandlung bei Dissoziation und Entwicklungs- oder Attachment-Trauma (Pierce 2014). Der theoretische Rahmen zum tanz-, bewegungstherapeutischen Drei-Phasen-Modell integriert dissoziierte somatische, emotionale und psychologische Erfahrungen und setzt sich aus folgenden Inhalten zusammen (Pierce 2014, 10, Übersetzung durch die Autorin):

\section{Phase 1: Sicherheit und Stabilisierung}

Funktion/Ziel: Unterstützen des empfundenen Sicherheitsgefühls durch interaktive Regulation, die innerhalb von Beziehungen entsteht

TT Interventionen:

- Körper-zu-Körper-Bezug durch Einstimmung (attunement) und Spiegeln

- Interaktive Regulation durch GroundingÜbungen und Orientierung im Hier und Jetzt

- 5-Sinnes-Wahrnehmung

- Einsatz von sensorischem Material

- Atemtechniken

\section{Phase 2: Integration von traumatischen Erinnerungen}

Funktion / Ziel: Pflegen eines verinnerlichten Sicherheitsgefühls, Ausweitung der Toleranz gegenüber inneren Erfahrungen

TT Interventionen:

- Aufmerksamkeit auf Interozeption und Selbstwahrnehmung verbindet die innewohnende Körperwahrnehmung mit Emotion und Kognition

- Symbolismus, Metapher, kreativer Ausdruck und künstlerische Distanz

\section{Phase 3: Entwicklung eines Beziehungs- Selbst und Rehabilitation}

Funktion / Ziel: Ermutigen zu Beziehungsfähigkeiten und Erweitern des Bewegungsrepertoires

TT Interventionen:

- Gruppensetting fördert Beziehung, Verbindung, Differenzierung und Bewegungsinteraktion

Das Modell orientiert sich am aktuellen Konsens des Best Practice Standards der phasenorientierten Behandlung. 


\section{Qualitative Analyse eines tanz-, bewegungstherapeutischen Projekts im Kontext eines Post-Konflikt- Entwicklungslandes}

Die explorative qualitative Studie von Dunphy et al. (2014) untersuchte ein zweitägiges tanz-, bewegungstherapeutisches Einführungsprogramm für traumatisierte und vertriebene Menschen im Post-Konflikt-Gebiet Timor-Leste (Dunphy et al. 2014). Der Workshop beabsichtigte, ein Vehikel für Themen zu Gesundheit und Wohlbefinden, Friedensschaffung, Erholung vom Trauma, kreatives Engagement und integrierte Körperwahrnehmungs-, Grounding-, Zentrierungs- und Ausdrucksübungen zu werden. Die qualitative Analyse der halbstrukturierten Interviews wies auf die bedeutende Rolle des kulturellen Tanzes und andere traditionelle Ausdrucksweisen für die Traumaverarbeitung hin. Gleichzeitig wurde die Notwendigkeit der Nachhaltigkeit eines solchen Programms betont. Die Anwendung des tanz-, bewegungstherapeutischen Programms wurde für diesen Fall als nicht sinnvoll eingestuft, da der fachliche Input und die Weiterentwicklung des Angebots im Anschluss nicht garantiert und finanziert werden konnte (Dunphy et al. 2014).

\section{Schlussfolgerung}

Die tanz-, bewegungs- und kreativtherapeutischen Interventionen bei Trauma und Dissoziation bei Kindern und Erwachsenen erfüllen bislang nicht die strengen Prüfungsstandards zur Evidenzbasierung, da große randomisierte Studien mit Behandlungstreue nach Protokoll und Handbüchern fehlen (Coleman/Macintosh 2015). Van Westrhenen und Fritz (2014) teilen die Kritik an qualitativen Studiendesigns und bewerteten deren Zuverlässigkeit als eingeschränkt. Die erlebnisund ausdrucksorientierten Ansätze bieten dennoch wichtige Ergänzungen und „Stand-alone-Interventionen“ zu evidenzbasierten Verfahren, um den komplexen Bedürfnissen traumatisierter Kinder und Erwachsener gerecht zu werden. Diese
Ansätze ermöglichen, dass traumatische Erinnerungen in eine Symbolsprache übersetzt werden, mit deren Hilfe emotionale Distanzierung einsetzen kann. Ohne überwältigt zu werden, können Teilnehmerlnnen Sicherheit, Stabilität, Vertrauen und beglückende Erfahrungen erleben. Spaltungen können aufgelöst und Gefühle sowie Schmerzen ausgedrückt werden (Sack/Sachsse 2013).

Eine engere Zusammenarbeit zwischen Forschung und Anwendung ist wünschenswert und notwendig (Levine/Land 2015). Die evidenzbasierte, tanz-, bewegungstherapeutische und körperpsychotherapeutische Praxis der Traumabehandlung sollte durch einen höheren Forschungsstandard überprüft werden. Denn positive Forschungsergebnisse auf hohem wissenschaftlichem Niveau können helfen, zukünftig Kreative Therapie und Tanz-, Bewegungstherapie als fundierte Ansätze für die Traumabehandlung zu etablieren.

\section{Literatur}

Coleman, K., Macintosh, H. B. (2015): Art and evidence: Balancing the discussion on arts- and evidence-based practices with traumatized children. Journal of Child \& Adolescent Trauma 8 (1), 21-31, http://dx.doi.org/10.1007/s40653-015-0036-1

Dunphy, K., Elton, M., Jordan, A. (2014): Exploring dance / movement therapy in post-conflict TimorLeste. American Journal of Dance Therapy 36(2), 189-208, http://dx.doi.org/10.1007/s10465-0149175-4

Jorba-Galdos, L. (2014): Creativity and dissociation. Dance / movement therapy interventions for the treatment of compartmentalized dissociation. The Arts in Psychotherapy 41 (5), 467-477, http:// dx.doi.org/10.1016/j.aip.2014.09.003

Levine, B., Land, H. M. (2015): A meta-synthesis of qualitative findings about dance / movement therapy for individuals with trauma. Qualitative Health Research (e-publ. ahead of print)

Pierce, L. (2014): The integrative power of dance / movement therapy: Implications for the treatment of dissociation and developmental trauma. The Arts in Psychotherapy 41(1), 7-15, http://dx.doi. org/10.1016/j.aip.2013.10.002

Sack, M., Sachsse, U. (2013): Therapiemethoden und Behandlungstechniken. In: Sack, M., Sachsse, U., Schellong, J. (Hrsg.): Komplexe Traumafolgestö- 
rungen: Diagnostik und Behandlung von Folgen schwerer Gewalt und Vernachlässigung. Schattauer, Stuttgart, 247-297

Spitzer, C., Wibisono, D., Freyberger, H.J. (2015): Theorien zum Verständnis von Dissoziation. In: Seidler, G. H., Freyberger, H. J., Maercker, A. (Hrsg.): Hand- buch der Psychotraumatologie. Klett-Cotta, Stuttgart, 22-37

Westrhenen, N. van, Fritz, E. (2014): Creative arts therapy as treatment for child trauma: An overview. The Arts in Psychotherapy 41(5), 527-534, http:// dx.doi.org/10.1016/j.aip.2014.10.004

\section{Die Autorin}

\section{Dr. Iris Bräuninger}

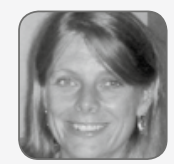

Wissenschaftliche Mitarbeiterin Psychiatrische Universitätsklinik Zürich, Dozentin im Masterstudiengang Tanztherapie, Autonome Universität Barcelona, Supervisorin, Ausbilderin und Lehrtherapeutin des deutschen und spanischen Berufsverbandes (BTD, ADMTE), Kestenberg Bewegungsanalytikerin, Psychotherapie (ECP), Private Praxis für Supervision und Therapie.
Dr. Iris Bräuninger

Wissenschaftliche Mitarbeiterin

Psychiatrische Universitätsklinik Zürich

Direktion Pflege, Therapien und Soziale Arbeit

Forschung und Entwicklung

Lenggstr. 31 | $\mathrm{CH}-8032$ Zürich

Tel. o041-(0)443842717

0041-(0)774422676

iris.braeuninger@puk.zh.ch oder dancetherapy@mac.com 